\title{
Численная модель формирования напряжённого состояния Земной коры вдоль профиля SVEKA за счёт денудации
}

\author{
Мягков Д.С. \\ ИФ3 РАН, Москва,dsm@ifz.ru
}

Аннотация. В работе рассматривается вопрос о физическом механизме, ответственном за формирование аномальных напряжений горизонтального сжатия в верхней части Земной коры, в том числе на Балтийском щите. В качестве такого механизма может выступать переход вещества верхней части Земной коры из закритического состояния за счёт денудации. Исследование ведётся методом численного моделирования. Рассматривается литосфера Балтийского щита вдоль профиля SVEKA. В рамках текущего исследования создана её геомеханическая модель, для которой методами численного моделирования рассчитано напряжённодеформированное состояние.

Ключевые слова: денудация, геодинамика, математическое моделирование, геомеханика.

\section{Numerical model forming the stressed state of the Earth's crustalong the SVEKA profile due to denudation processes}

\author{
Myagkov D.S. \\ IPERAS, Moscow,dsm@ifz.ru
}

\begin{abstract}
The article reviews the question of the physical mechanism responsible for the formation of anomalous horizontal compression stresses in the upper part of the Earth's crust, including on the Baltic Shield. Such a mechanism may be the transition of the substance of the upper part of the Earth's crust from the supercritical state due to denudation. The study is conducted by numerical simulation. The lithosphere of the Baltic Shield along the SVEKA profile is considered. As part of the current study, its geomechanical model was created, for which the stressstrain state was calculated by numerical simulation methods.
\end{abstract}

Key words: denudation, geodynamics, mathematical modeling, geomechanics.

\section{введение}

В данной работе исследуется проблема поиска геодинамического процесса, ответственного за формирование наблюдаемого в настоящее время напряжённого состояния Балтийского щита. Особенностью региона является Наличие достаточно большого количества геофизических данных, в первую очередь вдоль профиля SVEKA. В рамках текущего исследования предпринимается попытка поиска геодинамического объяснения данной закономерности методами численного моделирования. Создана численная двумерная модель, профиль которой сонаправлен со SVEKA. Для моделирования используется конечно-разностная схема Уилкинса, усовершенствованная Ю.П. Стефановым для применения в задачах геомеханики.

\section{Создание геомеханической численной модели}

Наличие достаточно большого количества геофизических данных вдоль профиля SVEKA (сейсмических, гравиметрических, реологических) (Varentsov et al., 2002; Korja et al., 2006; Glaznev, 2003; Moisio \& Kaikkonen, 2004] позволяет создать модель напряженно-деформированного состояния литосферы. Общая структура стартовой модели, сформированная на основе имеющихся геофизических данных (Glaznev, 2003), представлена ниже на рисунке 1. Высота модели - 70 км, длина - 850 км, ориентация - с юго-востока на северо-запад (по профилю SVEKA). Границы между основными телами (слоями) модели основаны на сейсмических данных (Luosto, 1984).

Основные тела (слои) модели и их средние параметры приведены в таблице 1. Земная кора на модели делится на три основных структурных элемента: гранитно-метаморфический слой (верхняя кора), гранулито-базитовый (средняя кора) и отдельный переходный слой нижней коры. Мантийная литосфера вводится отдельным элементом. Плотность слоев подбиралась с использованием грави- 
метрических данных (Glaznev et al., 1983). В таблице 1 показана средняя плотность, исключая небольшие отклонения от боковых средних значений (аналогично для скоростей $\mathrm{P}$ и $\mathrm{S}-$ волн).

Таблица 1. Средние значения параметров по модельным слоям.

Table 1. Average values of parameters for model layers.

\begin{tabular}{|c|c|c|c|c|}
\hline Слой & $\begin{array}{c}\text { Гранитно- } \\
\text { метаморфический }\end{array}$ & $\begin{array}{l}\text { Гранулито- } \\
\text { базитовый }\end{array}$ & $\begin{array}{c}\text { Переходный } \\
\text { слой }\end{array}$ & $\begin{array}{c}\text { Верхняя } \\
\text { мантия }\end{array}$ \\
\hline 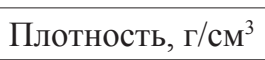 & 2.79 & 2.98 & 3.11 & 3.32 \\
\hline $\mathrm{V}_{\mathrm{p}}$, Kм/c & 6.20 & 6.73 & 7.51 & 8.10 \\
\hline $\mathrm{V}_{\mathrm{s}}, \mathrm{KM} / \mathrm{c}$ & 3.58 & 3.85 & 4.34 & 4.68 \\
\hline $\mathrm{k} \alpha$ & 0.05 & 0.10 & 0.12 & 0 \\
\hline Когезия, бар & 16 & 31 & 38 & 50 \\
\hline
\end{tabular}

Значения когезии и коэффициента угла внутреннего трения приведены в таблице 1 с учетом данных по тепловому полю, по геоэлектрическому разрезу (Korja et al., 2002; Korya, Lahti, Pedersen, 2006; Zhamaletdinov, 2011, Zhamaletdinov, Petrishchev, 2015) и относительно часто используемых значений в аналогичных численных моделях (Rebetsky et al., 2018). Коэффициент угла внутреннего трения был эффективно оценен с учетом влияния жидкости. Мантия рассматривалась как тело с законом пластичности Мизеса, соответственно коэффициент угла внутреннего трения принимался равным нулю.

Модель на рисунке 1 состоит из идентичных ячеек размером $500 \times 500$ м, общее количество ячеек составляет 238000 . Тела модели рассматривались как упрочняющиеся упруго-пластичные с законом пластического течения Друкера-Прагера-Николаевского для коры и Мизеса для мантии (Drukker \& Prager, 1975; Nikolaevsky, 1972). Для коровой части помимо параметров, указанных в таблице 1, введен параметр «коэффициент дилатансии», равный 0.3 (Nikolaevsky, 1972). Наличие ледниковых изостатических процессов подъема, который продолжается до настоящего времени, является главной особенностью земной коры в рассматриваемой области. Граница Мохо в центральной части профиля (в области 300-400 км) опускается до глубины 55-60 км со средними значениями 44 км на юго-западе и 38 км на северо-востоке (Pavlenkova, 2006, Sharov \& Mitriofanov, 2014). Следует отметить, что перепад высоты рельефа на профиле SVAKA составляет около 200 м, что определяет наличие пониженных значений давления в центральной части модели на рисунке 1.

\section{Результаты моделирования}

Напряженно-деформированное состояние модели, которая формируется под действием силы тяжести, было рассчитано на основе выбранных параметров и геометрии, сформированной на рисунке 1. Полученные литостатические напряжения преобладают по величине (за исключением поверхностного слоя модели), и они могут быть дополнены аномальными напряжениями тектонического характера. Метод Уилкинса (Wilkins, 1972), модифицированный для использования в геомеханике в (Stefanov, 2005), был выбран в качестве метода моделирования. Используется численная явная конечно-разностная схема. Задача решается при постановке механики твердого вещества и в двумерном виде (тип напряженного состояния - плоская деформация, промежуточная ось основного напряжения всегда перпендикулярна плоскости профиля). Результаты моделирования воздействия денудационных процессов представлены на рисунке 2.

Верхняя часть (рис. 1) показывает структуру напряженного состояния в виде отношения вертикального и горизонтального нормального напряжения (которое в двумерной формулировке характеризует положение основных осей). За исключением верхнего 3-километрового слоя, нижняя граница которого условно совпадает с кровлей слоя DD, модель находится в сверхкритическом состоянии, и рассматриваемое соотношение определяется как упругой плотностью, так и прочностными свойствами (прежде всего, коэффициентом угла внутреннего трения). В мантии с угловым коэффициентом, равным нулю, соотношение рассматриваемых компонентов регулярно получается равным единице. Земная кора находится в режиме вертикального сжатия, соотношение вертикальных 
и боковых напряжений в ней возрастает с 1.07 до 1.2. Локальные зоны горизонтального сжатия наблюдаются в некоторых случаях под действием только массовых сил в зонах градиентного рельефа (Rebetsky et al., 2018). В этом случае вся земная кора находится в состоянии вертикального сжатия. Нижняя часть (рис. 1) показывает интенсивность касательных напряжений в форме второго инварианта тензора напряжений. После введение процесса денудации (амплитуда - 600 м) в Верхней коре формируются отчётливо прослеживаемые области преобладания горизонтальных нормальных напряжений над вертикальными (рис. 2). Данная ситуация является достаточно характерной для Балтийского щита.

a

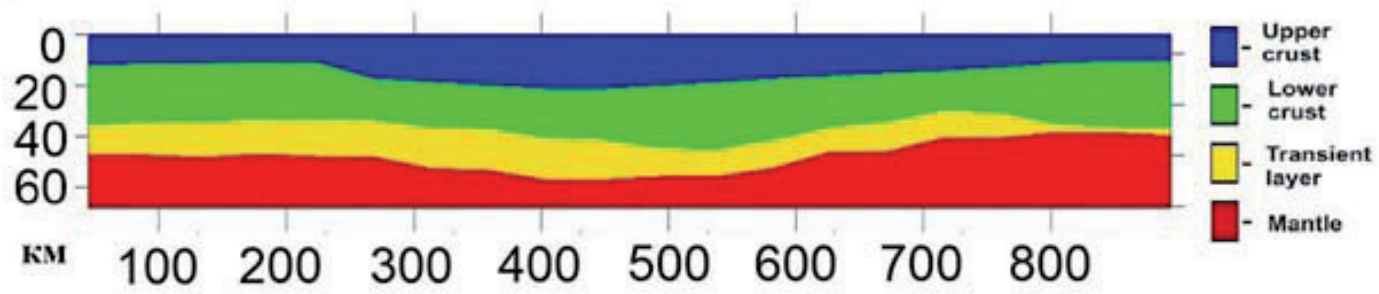

b

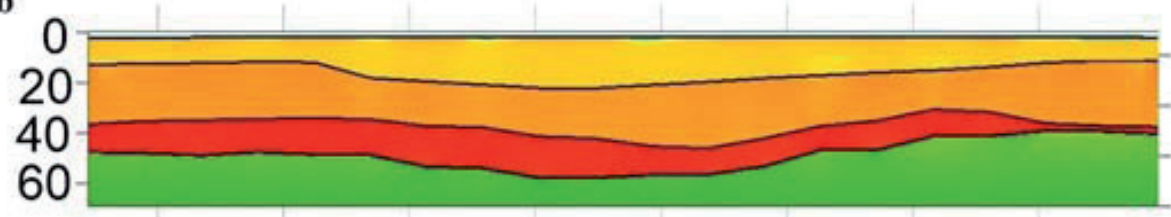

km $100 \quad 200 \quad 300 \quad 400 \quad 500 \quad 600 \quad 700 \quad 800$

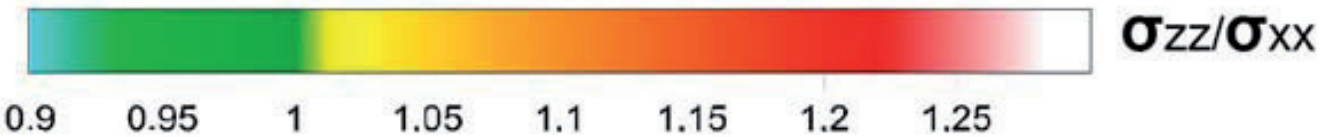

c
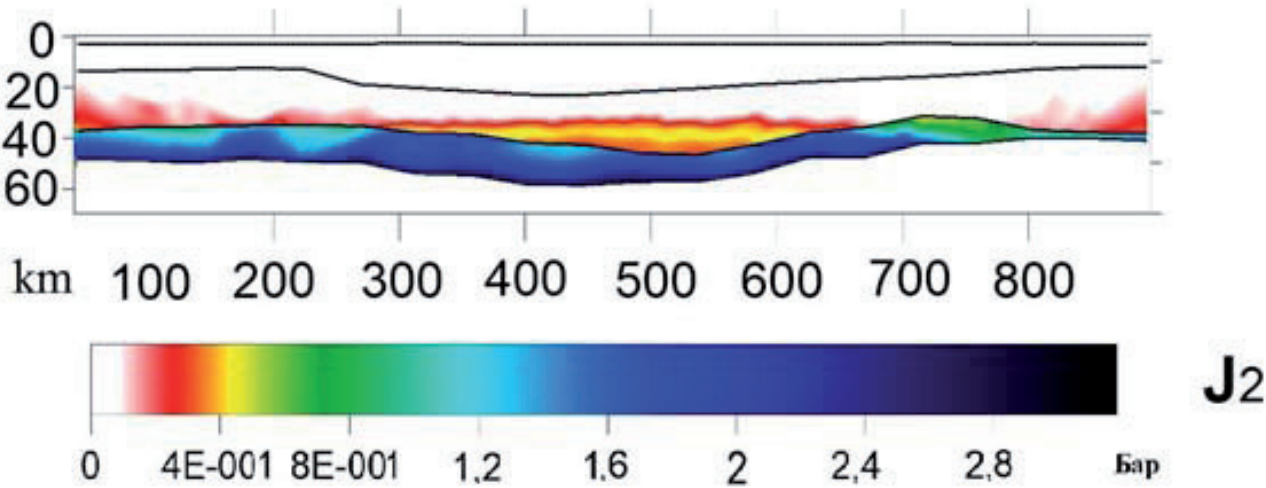

Рис. 1. (а) Общая геометрия стартовой модели, сформированная путем суммирования петрофизических данных (Glaznev, 2003) и результатов сейсморазведки (Korhonen \& Porkka, 1981), (b) отношение вертикальной и горизонтальной нормалей напряжения в модели (результаты моделирования под нагрузкой силами гравитации) и (с) максимальные сдвиговые напряжения в земной коре модели (в виде второго инварианта тензора напряжений до степени 0.5).

Fig. 1. (a) The general geometry of the launch model formed by summarizing the geophysical data (Glaznev, 2003) and the results of seismic exploration (Korhonen \& Porkka, 1981), (b) the ratio of vertical and horizontal normal stresses in the model (modeling results under load by mass gravitational forces) and (c) the maximal shear stresses in the core (in the form of the second invariant of the stress tensor to a power of 0.5). 


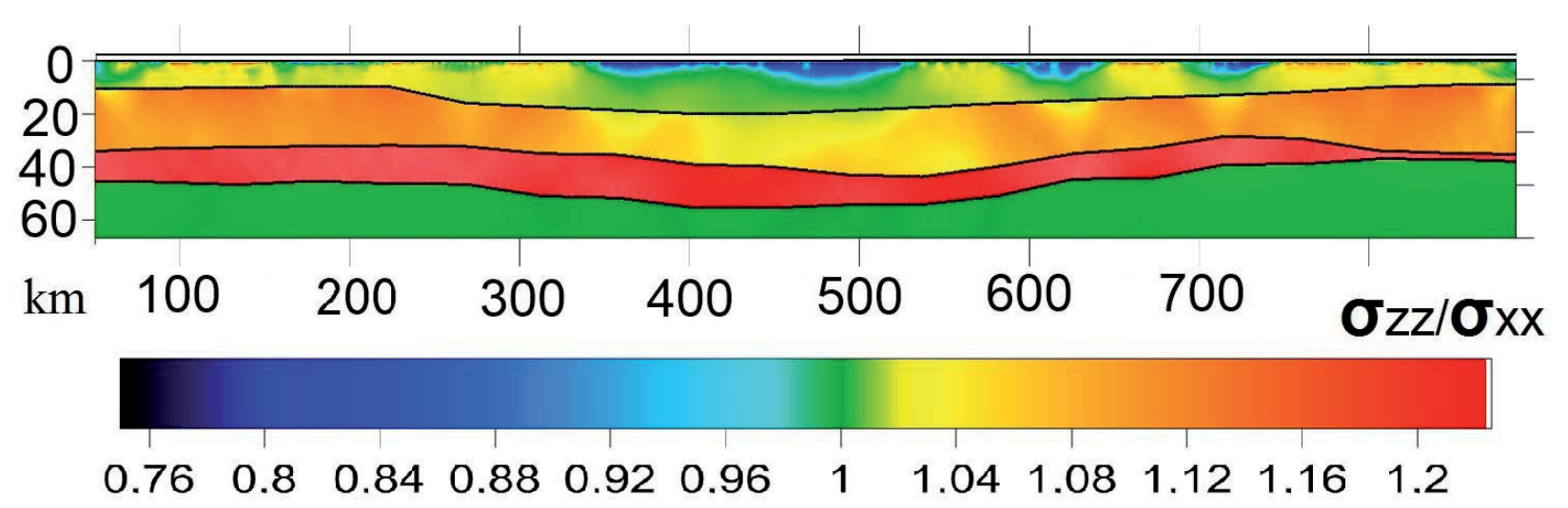

Рис. 2. Отношение вертикальной и горизонтальной нормалей напряжения в модели (результаты моделирования влияния денудации).

Fig. 2. The ratio of vertical and horizontal normal stresses in the model (modeling results of denudation effects).

В случае напряженного гравитационного состояния максимальные величины касательных напряжений наблюдаются в зонах контрастного рельефа в нижней части земной коры. В этом случае эти зоны легко прослеживаются в интервале 250-450 км. Величина интенсивности касательных напряжений при этом достигает 3 Бар. Отметим, что эта область максимального градиента касательных напряжений согласуется с широкой зоной поднятия (от 300 до 250 км) мантийного проводящего слоя, предположительно связанного с астеносферой, в интервале 200-400 км.

Созданная модель является достаточно обобщенной и отражает основные (литостатические) напряжения земной коры по профилю SVEKA и закономерности распределения параметров прочности. Это допускает дальнейшие модификации путем введения геодинамического процесса, который формирует дополнительный аномальный компонент напряженно-деформированного состояния. Воздействие от оси распространения, влияние процесса гляцио-изостазии и денудационно-накопительного воздействия рассматриваются как возможные источники модификации геодинамических процессов. Тем не менее, даже в существующей формулировке корреляция ряда структурных особенностей в напряженном состоянии и в разрезе удельного сопротивления позволяет проанализировать результаты интерпретации геоэлектрических данных с использование тектонофизического подхода.

\section{Выводы}

Рассмотрены элементы геодинамического анализа строения земной коры по профилю SVEKA. В случае напряженного гравитационного состояния максимальные величины касательных напряжений наблюдаются в зонах контрастного рельефа в нижней части земной коры. Эти зоны легко прослеживаются в интервале пикетов 250-450 км профиля SVEKA. Величина интенсивности касательных напряжений составляет около 3 Бар. Отметим, что эта область максимального градиента касательных напряжений согласуется с широкой, но довольно слабой зоной поднятия (от 300 до 250-280 км) мантийного проводящего слоя, предположительно связанного с астеносферой. Корреляция ряда структурных особенностей в напряженном состоянии и в разрезе удельного сопротивления литосферы позволяет считать перспактивным направлениес исследований путем интерпретации геоэлектрических данных с использованием тектонофизического подхода. Показано ярко выраженное преобладание аномальных напряжений в верхней части коры в области сноса геоматериала при достаточной амплитуде денудации (в среднем от 0.5 км). Это доказывает, что данный механизм генерации напряжений должен рассматриваться как один из ведущих, ответственных за формирования напряжённо-деформированного состояния в континентальной коре в геодинамических моделях, наряду с «классическими» эндогенными механизмами и должен рассматриваться в качестве самостоятельного источника формирования напряжённо-деформированного состояния в геодинамических моделях. 
Работа выполнена при финансовой поддержке РФФИ, проект № 18-05-00528, а также в рамках госзадания Министерства образования и науки РФ - тема ГИ КНЦ РАН № 0226-2019-0052 и темы ЦЭС КНЦ РАН № 0226-2019-0067.

\section{Литература}

1. Николаевский В.Н. Механические свойства грунтов и теория пластичности // Механика твёрдых деформируемых тел. Т. 6. Итоги науки и техники. М. Изд-во: ВИНИТИ АН СССР. 1972. С. 5-81.

2. Ребецкий Ю.Л., Погорелов В.В., Мягков Д.С., Ермаков В.А. О генезисе напряжений в коре островной дуги по результатам численного моделирования // Вестник КРАУНЦ. 2018. № 3. С. 54-73.

3. Ребецкий Ю.Л, Сим Л.А., Козырев А.А. О возможном механизме генерации избыточного горизонтального сжатия рудных узлов Кольского полуострова (Хибины, Ловозеро, Ковдор) // Геология рудных месторождений. 2017. Т. 59. № 4. С. 263-280.

4. Стефанов Ю.П. Некоторые особенности численного моделирования поведения упругохрупкопластичных материалов // Физ. мезомех. 2005. Т. 8. № 3. С. 129-142.

5. У Улккинс М.Л. Расчёт упруго-пластических течений // Вычислительные методы в гидродинамике. 1967. М. Изд-во: Мир. С. 212-263.

6. Glaznev V.N. Complex geophysical models of a lithosphere of the Fennoscandian. Apatity. «KaeM». 2003. $252 \mathrm{p}$.

7. Pavlenkova N.I. Structure of the lithosphere of the Baltic Shield according to the DSS / Struktura litosfery Baltiiskogo Shchita po dannym GSZ. Structure and Dynamics of the Lithosphere of Eastern Europe. Moscow. 2006. Geokart, GEOS. (in Russian).

8. Korja T. The BEAR Working Group. Lithosphere. Program and Extended Abstracts (Inst. of Seismology, Univ.of Helsinki, Helsinki), Report S-41. 2000.

9. Korja T. Lahti I. and Pedersen L. The core conductive structure along the SVEKA profile in the central part of the Baltic Shield // In the book: The structure and dynamics of Eastern Europe. Release 2. Moscow. Geocards Geos. 2006. P. 113-121.

10. Sharov N.V. and F.P. Mitrofanov. High-speed heterogeneity of the lithosphere of the Fennoscandian (Baltic) shield / Skorostnye neodnorodnosti litosfery Fennoskandinavskogo Shchita. Reports of the Academy of Sciences. 2014. 454 (2). P. 221-224. (in Russian).

11. Varentsov Iv.M., Engels M., Korja T., Smirnov M.Yu. and the BEAR Working Group. 2002. The generalized geoelectric model of Fehnnoscandia: a challenging database for long period 3D modeling studies within Baltic electromagnetic array research (BEAR) // Fizika Zemli. N. 10. P. 64-105.

12. Zhamaletdinov A.A. \& M.S. Petrishchev. Three_Dimensional Model of ithosphere Electrical Conductivity of the Fennoscandian Shield Based on the Results.of the BEAR and FENICS Experiments. ISSN 1028_334X, Doklady Earth Sciences. 2015. V. 463. Part 1. P. 751-756. C Pleiades Publishing, Ltd., 2015.Original Russian Text (C) A.A. Zhamaletdinov, M.S. Petrishchev. Published in Doklady Akademii Nauk. 2015. V. 463. N. 3. P. 333-338.

13. Zhamaletdinov A.A. The New Data on the Structure of the Continental Earth crust Based on the Results of Electromagnetic with the Use of Powerful Controlled Soures // Doklady Earth Sciences. 2011. V. 438. Part 2. P. $798-802$. 